Recepción: 07 / 04 / 2018

Aceptación: 09 / 06 / 2018

Publicación: 02 / 07 / 2018

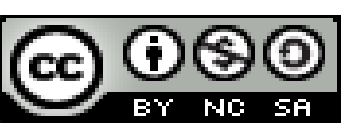

Ciencias de la educación

\title{
La calidad del servicio en las instituciones particulares de educación básica
}

\author{
The quality of the service in the special institutions of basic education
}

\section{A qualidade do serviço nas instituições particulares do ensino básico}

\author{
César A. Guerrero-Velástegui I \\ ca.guerrero@uta.edu.ec \\ Geovanny F. Ríos-Lara ${ }^{\mathrm{II}}$ \\ geovannyfrios@uta.edu.ec \\ Walter R. Jiménez-Silva ${ }^{\text {III }}$ \\ walterrjimenez@uta.edu.ec
}

Correspondencia: ca.guerrero@uta.edu.ec

\begin{abstract}
${ }^{\text {I } M a g i s t e r ~ e n ~ A d m i n i s t r a c i o ́ n ~ d e ~ E m p r e s a s ~ M e n c i o ́ n ~ P l a n e a c i o ́ n, ~ A b o g a d o ~ d e ~ l o s ~ T r i b u n a l e s ~ y ~ J u z g a d o s ~ d e ~ l a ~ R e p u ́ b l i c a ~}$ del Ecuador, Ingeniero de Empresas, Universidad de Zulia, Maracaibo, Venezuela.

${ }^{\text {II }}$ Magister Ejecutivo en Dirección de Empresas con Énfasis en Gerencia Estratégica, Diplomado Superior en Gerencia de Marketing, Diploma Superior en Gestión Prospectiva de la Educación, Especialista en Diseño Curricular, Especialista en Gerencia de Proyectos, Ingeniero en Empresas y Administración de Negocios, Licenciado en Ciencias Administrativas Administrador de Empresas, Universidad Técnica de Ambato, Ambato, Ecuador.

III Magister en Gestión Estratégica Empresarial Mba., Doctor en Ciencias Administrativas, Licenciado en Ciencias Administrativas Administrador Público, Universidad Técnica de Ambato, Ambato, Ecuador.
\end{abstract}




\title{
Resumen
}

La preocupación por la calidad del servicio en las instituciones de instituciones de Educación Básica Particulares en el Ecuador es fundamental e indispensable para el desarrollo de las actividades dentro de una sociedad. En este estudio se evaluó la calidad del servicio y el nivel de satisfacción de los clientes internos de las escuelas básicas particulares. El estudio fue de tipo descriptivo y como método de uso el inductivo-deductivo. Se utilizó como técnica la encuesta, y como instrumento un cuestionario constituido por 23 ítems. La validación del instrumento, se hizo a través del juicio de expertos y el cálculo de la confiabilidad por medio de índice de coeficiente de Alfa de Cronbach. La población de estudio fue de 43 colaboradores. Los resultados del análisis confirmaron la existencia de una correlación positiva media con un valor promedio de 0,694. Entre sus conclusiones, se constató que los factores relacionados con las inspecciones internas, las evaluaciones, retroalimentación y solución de problemas afectan indistintamente a las variables calidad del servicio (VI) y nivel de satisfacción de los clientes (VD).

Palabras clave: calidad del servicio; instituciones particulares; nivel de satisfacción; educación.

\begin{abstract}
The concern for quality of service in the institutions of the Ecuador private basic education institutions is fundamental and indispensable for the development of activities within a society. This study evaluated the quality of the service and the level of satisfaction of internal customers of private basic schools. The study was descriptive and as a method of using the inductivo-deductivo. Use it as a technical survey and as an instrument a questionnaire consisting of 23 items. The validation of the instrument, was made through expert opinion and the calculation of reliability using Cronbach's alpha coefficient index. The study population was 43 partners. The results of the analysis confirmed the existence of a positive correlation with an average value of 0,694. Among its conclusions, it was found that factors related to internal inspections, evaluations, feedback and troubleshooting affect both variables quality of service (VI), and level of satisfaction of clients (VD).
\end{abstract}

Keywords: quality of service; private institutions; level of satisfaction; education. 


\section{Resumo}

A preocupação com a qualidade do serviço nas instituições das instituições de educação básica no Equador é fundamental e indispensável para o desenvolvimento de atividades dentro de uma sociedade. Neste estudo, a qualidade do serviço e o nível de satisfação dos clientes internos das escolas básicas particulares foram avaliados. O estudo foi do tipo descritivo e como método indutivo-dedutivo. Utilizo como técnica o levantamento e como ferramenta um questionário composto por 23 itens. A validação do instrumento foi realizada por meio de julgamento pericial e cálculo de confiabilidade por meio do índice de coeficiente Alfa de Cronbach. A população do estudo foi de 43 colaboradores. Os resultados da análise confirmaram a existência de uma correlação positiva média com um valor médio de 0,694. Entre as suas conclusões, verificou-se que fatores relacionados com as inspecções internas, avaliações, feedback e resolução de problemas alternadamente variáveis que afetam a qualidade do serviço (VI) e nível de satisfação do cliente (VD).

Palavras chave: qualidade de serviço; instituições particulares; nível de satisfação; educação.

\section{Introducción}

En la actualidad, los servicios educativos en el mundo se han incrementado debido al crecimiento poblacional constante. Esto se debe al pro desarrollo generado por los países mayoritariamente de América del Sur. Las instituciones de Educación particulares han tenido que acogerse a diferentes estrategias que les permitan diferenciar su servicio, con lo cual han podido retener a los estudiantes, pero lastimosamente aquellas estrategias no han servido de mucho, pues al ser la sociedad constantemente cambiante existe la dificultad de poder llegar a cumplir con las exigencias y la satisfacción de la demanda educativa (Simancas, 2015).

En el caso particular del Ecuador, la mayor parte de las políticas de la calidad del servicio en las instituciones de educación es responsabilidad directa del Ministerio de Educación, de allí la necesidad que ha tenido el definir parámetros que se acoplen a las instituciones. Es así que, en las evaluaciones realizadas en el año 2010, la mayor parte de instituciones es decir el 60\% de instituciones públicas y privadas no cumplían con estándares de calidad, pero fueron consideradas que podrían dar un giro de $360^{\circ}$ para mejorar las dificultades, principalmente la pésima atención que se brindaban a los padres de familia al momento que se acercaban a conocer sobre el 
rendimiento que tenían sus hijos. Ello era aceptable en las públicas, pero en las particulares era muy diferente, pues por lo general no facilitaban las garantías necesarias para generar los servicios educativos. Pues la mayor parte de estas instituciones no contaban con instalaciones propias, los docentes no contaban con el nivel de preparación adecuado y sobre todo el perfil de ellos no era idóneo (Ministerio de Educación, 2015).

Según datos del Ministerio de Educación del Ecuador (2015), existen 569 instituciones Fiscomisionales, 248 municipales, 4559 particulares, dando un total de 2.687 en todo el país, las cuales se encuentran en un proceso de revisión de estándares de calidad en el servicio que componen muchos factores. Por ello, la importancia que representa el conocer el número de instituciones por provincia en Ecuador. Entre las que tienen el mayor número de instituciones educativas son Guayas, Manabí y Pichincha; la existencia de un mayor número de instituciones se debe a la densidad poblacional que existe en las provincias. En este caso, Manabí tiene 1.288 instituciones más que Pichincha, aunque, ésta última tenga el 16,4\% de población en edad escolar. En cuanto a la tasa de analfabetismo, Manabí tiene 9,1\% y Pichincha 3,8\% respectivamente. Mientras tanto, Galápagos es la provincia con un número menor de instituciones educativas, estas instituciones son las necesarias para satisfacer la demanda educativa en dicha provincia (posee la menor población estudiantil a nivel nacional).

Las primeras mediciones en la calidad del servicio se realizaron en la década de los noventa. El modelo ‘Aprendo' fue un paso relevante, cuando se valoró a los estudiantes a través de una muestra representativa, en Lenguaje y Matemática. Los resultados fueron negativos, debido a causas estructurales: la falta de procesos de desarrollo de la atención a los estudiantes y padres de familia; la ineficiente formación de los docentes y la evidente desarticulación de políticas y estrategias que vinculen al desarrollo de las actividades.

En la Provincia de Tungurahua, según el presupuesto del Ministerio de Educación, para el año 2014 el incremento fue de 2 veces más en comparación del año 2006, lo cual significó el incremento del $65 \%$ en la tasa de matrícula de estudiantes en las instituciones públicas, y de un 5\% en las instituciones privadas, lo cual es evidente por la gran inversión por la educación pública. El número de establecimientos educativos tanto fiscales, municipales, Fiscomisionales, particular laico y particular religioso públicos, por cantones es: Cantón Ambato 63 establecimientos, Baños de Agua 
Santa 7, Cevallos 3, Mocha 3, Patate 7, Quero 7, San Pedro de Pelileo 16, Santiago de pillarlo 12 y Tisaleo 5; dando un total de 123 instituciones en la provincia de Tungurahua (Ministerio de Educación del Ecuador, 2015).

Las instituciones educativas presentan varios problemas en el aspecto de la gestión de las instituciones particulares, pues las instituciones al ser de personas particulares, por lo general se dedican a lucrar sin considerar el cumplimento de los parámetros mínimos. El problema central de las instituciones particulares es el desconocimiento de los atributos de calidad en el servicio, esto debido que se evidencia según los estudios realizados en la provincia que existe una atención a los padres de familia dada su insatisfacción, pues buscan que exista una comunicación apropiada con los docentes (Ministerio de Educación del Ecuador, 2015).

Por otra parte, a menudo se evidencia que en una minoría las instituciones particulares tienen una correcta presentación del personal docente, pues los docentes, aunque tienen los uniformes incumplen en el uso de los mismos porque por lo general no les gusta. Además, las instituciones no proyectan adecuadamente la imagen institucional por el uso incorrecto del logotipo que, por lo general, estos factores han disminuido el oleaje para el ingreso de nuevos estudiantes debido que no se han generados estrategias de integración de los procesos al ofertar los servicios educacionales. A tal efecto, en este artículo se evaluó la calidad del servicio y el nivel de satisfacción de los clientes internos de las escuelas básicas particulares.

\section{Desarrollo}

Los elementos que constituyen la calidad de la educación, en muchos estudios hay resultados inadecuados, específicamente en los procesos de prestación del servicio al cliente externo. Así tenemos, que en el estudio realizado en Barcelona sobre la educación fueron definidos otros criterios de calidad del servicio que se reflejan en todo lo que la organización hace, como se realiza y cómo se gestiona, entre los más importantes encontrados al evaluar la calidad están: el liderazgo en un $12 \%$, personas profesionales $7 \%$, planificación y estrategia $10 \%$, colaboradores y recursos $7 \%$ y procesos $14 \%$. En cuanto a los resultados que se pretenden alcanzar están clasificados en: resultados en personal $11 \%$, resultados en usuarios $15 \%$, resultados en entorno $10 \%$ y resultados clave $14 \%$ que son las ponderaciones dadas por los organismos de control de la educación en España (Folgueiras, Luna, y Puig, 2014). En este estudio, se suman los criterios que fueron 
considerados para medir la calidad del servicio en las instituciones de educación secundaria del Valle de Toluca, entre los más importantes se mencionan seis grupos de criterios para medir la calidad del servicio, los cuales tienen una ponderación cada uno: los métodos de enseñanza y evaluación (15\%) con una metodología aplicada, incorporación de las nuevas tecnologías y sistema de evaluación. El nivel de autorrealización del estudiante (15\%) constituido por las calificaciones o resultados obtenidos, conocimientos y habilidades adquiridas, valores adquiridos y capacitación para la inserción al mundo laboral. Los servicios de apoyo (15\%) que integra el servicio bibliotecario, acceso a sala de cómputo, acceso a internet, servicio de fotocopiado, cafetería escolar, servicio médico, acceso a actividades culturales y, artísticas y recreativas (Álvarez, Chaparro, \& Reyes, 2015).

Además, los servicios administrativos (15\%) que está integrado por control escolar y proceso de admisión e inscripción. El ambiente propicio (15\%) estructurado por la atención por parte del personal administrativo, atención del personal de servicios de apoyo y mantenimiento y la atención por parte del personal docente Ambiente estudiantil. Y la infraestructura (15\%) compuesta de la limpieza de las instalaciones, condiciones del mobiliario, espacios para la enseñanza (salones, talleres, laboratorios), espacios para el descanso y recreación, instalaciones de la biblioteca, instalaciones del área de cómputo, instalaciones de la cafetería escolar, instalaciones deportivas e instalaciones sanitarias (Álvarez, Chaparro, \& Reyes, 2015).

En este sentido, el autor expone que resulta difícil tratar de definir la calidad del servicio en la educación, lo cual se ha debatido por décadas y ha dado origen a considerar que es la promoción de las actitudes de las autoridades, docentes y personal administrativo; como la dimensión funcional en la prestación del servicio la que se relaciona con el tratamiento que reciben los estudiantes y padres de familia. Pero el cambio de milenio ha promovido variaciones drásticas por el aparecimiento de las tecnologías de la información y la competencia de mercados nacionales e internacionales. Pues el incremento de la demanda fue sustancial desde los años 70 de donde se desprendió varios paradigmas y teorías en la efectividad en la educación (Valenzuela, 2013).

\section{La calidad en el servicio educativo.}

La preocupación por la calidad en la educación, tiene una trascendencia emblemática y se remonta desde finales del siglo XVIII. La universalización de la evaluación de los aprendizajes, según los 
mismos criterios y prácticas, y el interés creciente de los estados por optar la educación de masas y dar cuerpo a sistemas educativos nacionales son dos expresiones de este hecho. Pero la problemática de la calidad de la educación, a raíz de las dinámicas culturales que en términos globales se desencadenan en las últimas décadas del siglo XX, adquiere una serie de matices particulares que la hacen más compleja y necesitada de un tratamiento en el que converjan dimensiones como la económica, política, filosófica, curricular y, por supuesto, la pedagógica (Orozco, Toro, \& Duarte, 2009).

En este sentido, casi la mitad de los países han introducido políticas para mejorar la formación profesional la mayoría de las políticas se han centrado en aumentar la calidad y algunos países han introducido nuevos cursos programas o la acreditación de programas para el control de calidad. En la calidad educativa actualmente en el ámbito educado se justifica con cualquier proceso de cambio o planes de mejora de la educación (Pont, 2015). Cuando se abusa tanto de un vocablo como el de calidad de la educación se imponen una serie de trampas terminológicas, intrínsecas y extrínsecas, cuya finalidad radica en presentar unas determinadas políticas y decisiones educativas como si fueran naturales, un resultado de fuerzas irreversibles, ligadas al mercado (Vega, 2014).

Cabe destacar, que la gestión de la calidad para las empresas tanto a nivel empresarial como académico, constituye una de las prioridades en su quehacer cotidiano, con vistas a garantizar un cierto nivel de competitividad que les permita sobrevivir en el mercado. Si no se garantiza que el producto o servicio final satisfaga las necesidades de los clientes con rapidez y al mínimo coste no se logrará dicho objetivo (Parra, Pavón, Guerrero, \& Suárez, 2016). Una gestión eficiente y eficaz de los recursos y problemas públicos, y de la atención a las necesidades esenciales de la sociedad representa un importante mecanismo de transformación social y forma la base de las economías exitosas y en la actualidad como un sistema de gestión, orientado a la calidad y a la reducción de costos (Sampaio \& Romero, 2017; González \& Moreno, 2016).

\section{Satisfacción de los usuarios en las unidades educativas}

Es de destacar, que la satisfacción del servicio surge de la calidad del servicio "término que data desde la década de 1990", cuyo sistema está definido con la misma rigurosidad que para la satisfacción del servicio. Se trata entonces, de un sistema con todas las consecuencias que esto acarrea, sobre todo, en lo que se refiere al rigor necesario en cuanto a la concepción y la puesta en 
funcionamiento. Pero, la gran diferencia en relación con la fabricación de un producto reside en el hecho de que el cliente forma parte integrante del sistema de calidad, él es uno de los actores, a la vez productor y consumidor. En relación a la definición que antecede se identifican entre los elementos que forman parte del sistema de servició: el cliente (consumidor implicado en la fabricación del servicio), el soporte físico (soporte material), el personal de contacto (persona en contacto, directo con el cliente), el servicio (este resultado constituye el beneficio que debe satisfacer la necesidad del cliente) el sistema de organización interna (no visible para el cliente, funciones clásicas de la empresa) y por último los demás clientes (intercambio de comunicación entre) (Ríos , 2014).

\section{Materiales y métodos}

El estudio se enmarcó en una investigación de tipo descriptiva, que conllevó a entender de mejor manera las causas y efectos del problema, e identificar las relaciones entre las variables de estudio. El enfoque epistémico utilizado fue el histórico lógico, el cual facilitó el entender, el origen, la calidad del servicio y el nivel de satisfacción de los clientes, a través del método inductivodeductivo. Además, para el levantamiento de la información se consideró utilizar la técnica de la encuesta a partir de un instrumento tipo cuestionario constituido por 23 ítems con opciones de respuesta de escala de Likert cuyas alternativas fueron entre nunca y siempre con la opción de abstenerse. La validación del instrumento, se hizo a través del juicio de expertos y el cálculo de la confiabilidad por medio de índice de coeficiente de Alfa de Cronbach. La población de estudio fue de 43 colaboradores de las Unidades Educativas Particulares del Cantón de Ambato, es decir a los rectores. Además, para la comprobación de las hipótesis se consideró el modelo estadístico del Coeficiente de Pearson para identificar las relaciones entre ambas variables de estudio (Triola, 2009; Fernández, Hernández, \& Baptista, 2010; Fidias, 2012). Para el procesamiento de la información y comprobación de las hipótesis, se empleó el software SPSS Statistics versión 23.0.

\section{Resultados y discusión}

Para el desarrollo del modelo se consideró como variable independiente (calidad de servicio) cuyas sub variables de medición fueron: gestión, indicadores, objetivos y ambiente. En cuanto a la variable dependiente (nivel de satisfacción), las sub variables planificación, inspecciones, actividades, satisfacción, logros, sesiones, evaluaciones, retroalimentación, trabajo en equipo, 
prevenir e intervienen. El procedimiento se realizó buscando la relación de cada sub variable, los resultados del análisis se presentan en la siguiente tabla.

Tabla 1. Correlaciones significativas

\begin{tabular}{|c|c|c|c|c|c|c|}
\hline & & Inspecciones & $\begin{array}{c}\text { Departamento } \\
\text { de talento }\end{array}$ & Indicadores & Seguimiento & Satisfacción \\
\hline \multirow[t]{3}{*}{ Inspecciones } & Correlación de Pearson & 1 &, $616^{* *}$ &, $880^{* *}$ &, $503^{* *}$ &, $878^{* *}$ \\
\hline & Sig. (bilateral) & & 000 &, 000 &, 001 &, 000 \\
\hline & $\mathrm{N}$ & 43 & 43 & 43 & 43 & 43 \\
\hline \multirow{3}{*}{$\begin{array}{l}\text { Departamento } \\
\text { de talento }\end{array}$} & Correlación de Pearson & & 1 &, $857^{* *}$ &, $635^{* *}$ &, $663^{* * *}$ \\
\hline & Sig. (bilateral) & & &, 000 &, 000 &, 000 \\
\hline & $\mathrm{N}$ & & 43 & 43 & 43 & 43 \\
\hline \multirow[t]{3}{*}{ Indicadores } & Correlación de Pearson & & & 1 &, $587^{* * *}$ &, $897^{\text {*** }}$ \\
\hline & Sig. (bilateral) & & & &, 000 &, 000 \\
\hline & $\mathrm{N}$ & & & 43 & 43 & 43 \\
\hline \multirow[t]{3}{*}{ Seguimiento } & Correlación de Pearson & & & & 1 &, $421^{* * *}$ \\
\hline & Sig. (bilateral) & & & & &, 005 \\
\hline & $\mathrm{N}$ & & & & 43 & 43 \\
\hline \multirow[t]{3}{*}{ Satisfacción } & Correlación de Pearson & & & & & 1 \\
\hline & Sig. (bilateral) & & & & & \\
\hline & $\mathrm{N}$ & & & & & 43 \\
\hline
\end{tabular}

Elaborado por: Santiago Sánchez **. La correlación es significativa en el nivel 0,01 (bilateral).

Fuente: Elaboración propia

En la Tabla 1, se aprecian las relaciones entre los diferentes factores que intervinieron en la evaluación de las variables en estudio; la calidad del servicio (VI) y el nivel de satisfacción de los clientes (VD), donde se presenta un promedio de 0,694 por lo cual se considera que existe una correlación positiva media.

Se puede denotar que la correlación de mayor influencia de la fila es entre inspección-indicadores con un índice de $(0,880)$, con lo cual se demuestra que existe una correlación positiva media. Pues las inspecciones permiten identificar si la educación es buena o mala, es decir identificar los problemas que existen y como mejorarlos (Menin, 2013).

Por otra parte, en la fila dos la correlación de mayor aporte fue entre departamento de talentoindicadores con un índice de $(0,857)$, con lo cual se demuestra que existe una correlación positiva media. Ello debido al cómo se desarrolló el talento humano y de ello dependerá mucho el cumplimiento de indicadores y logro de resultados (Pietro \& Delgado, 2010). 
En la tercera fila de las correlaciones se evidencia que la relación de mayor relevancia fue entre los indicadores -satisfacción con un índice de $(0,897)$, con lo cual se muestra una correlación positiva media. Pues los indicadores están ligados estrechamente con la satisfacción de los clientes. Esto sucede por la mercantilización de la educación, y la consecuente identificación de las instituciones educativas como organizaciones empresariales y de los estudiantes como consumidores (Alvaréz \& Chaparro, 2014).

En la cuarta fila de las correlaciones se demuestra que no es muy significativa entre el seguimientosatisfacción $(0,421)$, con lo cual se demuestra una correlación positiva considerable. Debido que si el seguimiento que se realice en la institución es inadecuado la satisfacción de los clientes se reflejara, es muy común porque las políticas del estado es garantizar la educación por ende en todo momento las instituciones mantienen un seguimiento de las actividades que realizan (González \& González, 2012).

Una vez revisado los valores calculados y los criterios de decisión, se pudo constatar que la calidad del servicio se relaciona con el nivel de satisfacción de los clientes en las Instituciones de Educación Básica Particulares”, obteniéndose una correlación positiva media (Visauta, 2007).

\section{Conclusiones}

En cuanto a las Unidades Educativas Básicas Particulares, el enfoque a la calidad esta dado en la formación profesional, en programas, control y acreditación institucionalmente, con el objetivo de crear procesos de cambios y planes de mejora de la educación que contribuyan en optimizar el nivel de satisfacción de los clientes.

Las cualidades del servicio se centran en la generación de políticas de mejora continua para los clientes externos en la educación, para ello se debe considerar e identificar ciertas características que la sociedad posee, en la cual la calidad es determinante en el nivel de satisfacción de los clientes.

Los factores vinculados en el nivel de satisfacción de los clientes están dados por las inspecciones que se realicen en los procesos, la planificación de los recursos y bienes, las actividades, logros, reuniones que se realizan, evaluaciones a los estudiantes, personal administrativo y académico, retroalimentación, trabajo en equipo y la prevención para no cometer errores innecesarios. Sin 
embargo, se evidenció que los factores evaluaciones, retroalimentación y solución de problemas son los que afectan en mayor grado a las instituciones estudiadas.

Se constató que los factores relacionados con las inspecciones internas, las evaluaciones, retroalimentación y solución de problemas afectan indistintamente a las variables calidad del servicio (VI) y nivel de satisfacción de los clientes (VD).

\section{Referencias Bibliográficas}

Álvarez, J., Chaparro, E., \& Reyes, D. (2015). Estudio de la Satisfacción de los Estudiantes con los Servicios Educativos brindados por Instituciones de Educación Superior del Valle de Toluca. Iberoamericana sobre Calidad, XIII(2), 5-26. doi:E-ISSN: 1696-4713

Fernández, C., Hernández, R., \& Baptista, M. (2010). Metodología de la Investigación (Quinta ed.). Distrito Federal, México: Mc Graw-Hill. doi:ISBN: 978-607-15-0291-9

Fidias, G. (2012). El proyecto de investigación ( $6^{\circ}$ ed.). Caracas, Venezuela: Episteme. doi:ISBN: 980-07-8529-9

Folgueiras, P., Luna, E., \& Puig, G. (2014). El aprendizaje y servico en educación secundaria. Iberoamericana de Educación, 1-15. doi:ISSN:1681-5653

González, L., \& Moreno, M. (2016). Procedimiento para implementación de un sistema de gestión de costos de calidad. Ciencias Holguín, XXII(2), 1-14. doi:E-ISSN: 1027-2127

González, O., \& González, L. (2012). Estilos de liderazgo del docente universitario. Multiciencias, XII(1), 35-44. doi:ISSN: 1317-2255

Menin, O. (2013). ¿Qué es una educación de calidad? Praxis Educativa (Arg), I(2), 47-50. doi:ISSN: 0328-9702

Ministerio de Educación. (2015). Ministerio de Educación. Obtenido de Estnadares de calidad educativa: https://educacion.gob.ec/wp-content/uploads/downloads/2013/03/estandares_2012.pdf

Ministerio de Educación. (2015). Ministerio de Educación. Obtenido de Estadsitica Educativa: https://educacion.gob.ec/wpcontent/uploads/downloads/2016/01/Publicaciones/PUB_EstadisticaEducativaVol1_mar2015.pdf 
Ministerio de Educación del Ecuador. (2015). Sistema integral de tecnologias para la escuela y la comunidad. Obtenido de Sistema integral de tecnologias para la escuela y la comunidad: https:/educacion.gob.ec/wp-content/uploads/downloads/2015/11/Proyecto_SITEC.pdf

Morales, D. (2017). Las concepciones de curriculum y su importancia en la elaboración de un doctorado curricular en estomatología. Cubana Estomatol, XXXXVIII(3), 301-314. doi: ISSN 0034-7507

Orozco, J., Toro, A., \& Duarte, V. (2009). La calidad de la educación o educación de calidad?una preocupacion mas alla. Iberoamericana de Educación (51), 161-181. doi:ISSN (Versión impresa): 1022-6508-X

Osorio, J. (2015). La calidad en la educación y la salud. CES Medicina, XXIX(1), 5-6. doi:ISSN: 0120-8705

Palacio, C. (2016). La calidad de los psiquiatras en Colombia. Colombiana de Psiquiatría, XXXXV(2), 59. doi:ISSN: 0034-7450

Parra, L., Pavón, Y., Guerrero, Y., \& Suárez, J. (2016). Procedimiento de un sistema de comunicación para la gestión de calidad. Ciencias Holguín, XXII(3), 1-17. doi:E-ISSN: 1027-2127

Pietro , G., \& Delgado, A. (2010). Fiabilidad y validez. Papeles del Psicólogo, XXXI(1), 67-74. doi:ISSN: 0214-7823

Ríos , L. (2014). Los servicos no son prodcutos: la servución y la importancia de su proceso en las empresas. Ventana Cientifica, IV(8), 55-67. doi:ISSN: 2305 - 6010

Sampaio, A., \& Romero, A. (2017). Modelo y procedimiento para la calidad de la gestión en municipios pequeños de Brasil. Ingeniería Industrial, XXXVIII(1), 93-105. doi:ISSN: 0258-5960

Simancas, R. (2015). La servucción como estrategia para la recuperación del servicio no prestado en las instituciones de educación superior. Colombiana de Marketing, XVIII(13), 42-49. doi:ISSN: $1657-4613$

Triola, M. (2009). Estadística (Décima ed.). México: Pearson Educación. 
Valenzuela, H. (2013). Los sistemas de calidad en las instituciones educativas tendnecias actuales. La educación (142), $\quad 1-28 . \quad$ Obtenido de http://www.educoea.org/portal/La_Educacion_Digital/laeducacion_142/articles/LosSistemasdeC alidadenlasInstitucionesEducativasTendenciasActuales_HugoValenzuela_1.pdf

Vega, R. (2014). La calidad educativa una noción neoliberal, propia del darwinismo pedagógico. Integra Educativa. Obtenido de http://www.scielo.org.bo/scielo.php?pid=S1997$40432014000200007 \&$ script=sci_arttext\&tlng=en

Visauta, B. (2007). Análisis estdístico con SPSS 14 (Tercera ed.). España: McGraw-Hill. doi:ISBN: 978-84-481-5670-1 